\title{
Fault Tolerant Control for Civil Structures Based on LMI Approach
}

\author{
Chunxu Qu, Linsheng Huo, and Hongnan Li \\ Faculty of Infrastructure Engineering, Dalian University of Technology, Dalian 116024, China \\ Correspondence should be addressed to Linsheng Huo; lshuo@dlut.edu.cn
}

Received 18 July 2013; Revised 22 September 2013; Accepted 16 October 2013

Academic Editor: Bo-Chao Zheng

Copyright (C) 2013 Chunxu Qu et al. This is an open access article distributed under the Creative Commons Attribution License, which permits unrestricted use, distribution, and reproduction in any medium, provided the original work is properly cited.

\begin{abstract}
The control system may lose the performance to suppress the structural vibration due to the faults in sensors or actuators. This paper designs the filter to perform the fault detection and isolation (FDI) and then reforms the control strategy to achieve the fault tolerant control (FTC). The dynamic equation of the structure with active mass damper (AMD) is first formulated. Then, an estimated system is built to transform the FDI filter design problem to the static gain optimization problem. The gain is designed to minimize the gap between the estimated system and the practical system, which can be calculated by linear matrix inequality (LMI) approach. The FDI filter is finally used to isolate the sensor faults and reform the FTC strategy. The efficiency of FDI and FTC is validated by the numerical simulation of a three-story structure with AMD system with the consideration of sensor faults. The results show that the proposed FDI filter can detect the sensor faults and FTC controller can effectively tolerate the faults and suppress the structural vibration.
\end{abstract}

\section{Introduction}

The structural control system is commonly classified into four types: passive, active, hybrid, and semiactive control [1-9]. An active control system contains sensors, controllers, and actuators. Sensors can measure the structural vibration data that is sent to the controllers. The controllers compute the desired force according to the control strategy and sensor data and then command the control devices to generate control force for vibration reduction. Active control can achieve many control decisions. Therefore, active control is an efficient method to resist external excitation, such as earthquake and strong wind. Control strategy is the key of active control, which is widely studied in recent decades [10-16]. However, there are some controller design problems in civil engineering due to the characteristics of the civil structures, such as large number of degrees of freedom and uncertainties of system parameters, in which many researchers are interested [17-21].

In practical engineering, sensors and actuators may be destroyed due to the lack of maintenance or extreme loading such as strong wind or intense earthquake. These faulty sensors and control devices may degrade the performance of vibration suppressing and even enlarge the structural response. Therefore, the robustness is important for the control system. Fault tolerant control (FTC) technology is an efficient method to promote the system reliability. FTC can tolerate faults caused by the components of control system, such as sensors and actuators, and then guarantee the system performance. FTC can be divided into two categories, passive FTC and active FTC. The difference between them exists if the faults are identified online. Passive FTC cannot detect faults online and cannot be recognized as traditional robust control [22]. The faults in passive FTC are assumed as system model uncertainties. Accordingly, passive FTC has limited tolerance ability because its robustness is only for the estimated faults, and the faults cannot be detected online. On the other hand, active FTC can detect faults online, which is called the fault detection and isolation (FDI) technology, and suppress excessive structural response.

This paper designs a fault detection and isolation (FDI) filter and reforms a fault tolerant controller (FTC) through LMI approach considering sensor faults in civil building structures with active mass damper (AMD). An estimated system is built to facilitate the design of FDI and FTC. In order to make the difference between the estimated and the practical system smaller, an FDI filter is designed, which 
can minimize the Ho norm of the transfer function from all the inputs to the residual of the estimated and practical state. According to the bounded real lemma, solving the FDI filter problem is transformed to solve a matrix inequality, which can be deformed to a linear matrix inequality (LMI) by variable substitution method [23]. LMI problem is convex and can be solved easily. After the FDI filtering, the new data is used to isolate the sensor faults and transmitted to the previous designed controllers so that the control strategy is reformed. The process executes the function of fault tolerant control (FTC). Finally, the numerical example of a three-story building is provided to validate the performance of FDI and FTC.

\section{Formulation of AMD Control System}

Considering $n$ degrees of freedom civil structural system which is subjected to a unidirectional earthquake with $\ddot{q}_{g}(t)$ acceleration, the dynamic equation is described as follows:

$$
\mathbf{M} \ddot{\mathbf{q}}(t)+\mathbf{C} \dot{\mathbf{q}}(t)+\mathbf{K q}(t)=\mathbf{T}_{g} \ddot{q}_{g}(t)+\mathbf{T}_{u} \mathbf{u}(t),
$$

where $\mathbf{M}, \mathbf{C}$, and $\mathbf{K} \in \mathbb{R}^{n \times n}$ are, respectively, mass, damping, and stiffness matrix; $\mathbf{q}(t), \dot{\mathbf{q}}(t)$ and $\ddot{\mathbf{q}}(t) \in \mathbb{R}^{n \times 1}$ are the relative displacement, relative velocity, and relative acceleration vector, respectively; $\mathbf{u}(t) \in \mathbb{R}^{m_{u} \times 1}$ is the control force vector generated by actuators and $\mathbf{T}_{u} \in \mathbb{R}^{n \times m_{u}}$ and $\mathbf{T}_{g} \in \mathbb{R}^{n \times 1}$ are the location matrices of the control force and earthquake excitation, respectively.

For a structure with an AMD on the top floor as shown in Figure 1, the control force is exerted only on the top floor. The control force location matrix $\mathbf{T}_{\mathcal{u}}$ and earthquake excitation location matrix $\mathbf{T}_{g}$ are represented as

$$
\begin{gathered}
\mathbf{T}_{u}=[\underbrace{0,0 \ldots, 0,}_{n-1} 1]_{1 \times n}^{T}, \\
\mathbf{T}_{g}=-\mathbf{M}\{\mathbf{1}\}_{n \times 1} .
\end{gathered}
$$

The dynamic equation of the AMD system can be formulated as

$$
\begin{gathered}
m_{a}\left(\ddot{q}_{a}(t)+\ddot{q}_{g}(t)\right)+c_{a}\left(\dot{q}_{a}(t)-\dot{q}_{n}(t)\right) \\
+k_{a}\left(q_{a}(t)-q_{n}(t)\right)=F_{a}(t),
\end{gathered}
$$

where $m_{a}, c_{a}$, and $k_{a} \in \mathbb{R}^{1 \times 1}$ are, respectively, mass, damping, and stiffness of AMD system and $q_{a}(t), \dot{q}_{a}(t)$, and $\ddot{q}_{a}(t) \epsilon$ $\mathbb{R}^{1 \times 1}$ are the relative displacement, relative velocity, and relative acceleration of AMD system. $q_{n}(t)$ and $\dot{q}_{n}(t) \in \mathbb{R}^{1 \times 1}$ are relative displacement and relative velocity of the $n$th floor, which is also the top floor and can be represented as

$$
\begin{gathered}
q_{n}(t)=\mathbf{T}_{n} \mathbf{q}(t), \quad \dot{q}_{n}(t)=\mathbf{T}_{n} \dot{\mathbf{q}}(t), \\
\mathbf{T}_{n}=[\underbrace{0,0 \ldots, 0,}_{n-1} 1]_{1 \times n} .
\end{gathered}
$$

$F_{a}(t) \in \mathbb{R}^{1 \times 1}$ is the force between structural system and AMD mass. The motion equation of the structural system with AMD can be derived as

$$
\mathbf{M}_{s} \ddot{\mathbf{q}}_{s}(t)+\mathbf{C}_{s} \dot{\mathbf{q}}_{s}(t)+\mathbf{K}_{s} \mathbf{q}_{s}(t)=\mathbf{T}_{g s} \ddot{q}_{g}(t)+\mathbf{T}_{u s} F_{a}(t) \text {. }
$$

The matrices $\mathbf{M}_{s}, \mathbf{C}_{s}, \mathbf{K}_{s}, \mathbf{q}_{s}, \mathbf{T}_{g s}$, and $\mathbf{T}_{u}$ are denoted as

$$
\begin{aligned}
& \mathbf{M}_{s}=\left[\begin{array}{ll}
\mathbf{M} & \\
& m_{a}
\end{array}\right]=\left[\begin{array}{llll}
m_{1} & & & \\
& \ddots & & \\
& & m_{n} & \\
& & & m_{a}
\end{array}\right]_{(n+1) \times(n+1)}, \\
& \mathbf{q}_{s}=\left[\begin{array}{c}
\mathbf{q} \\
q_{a}
\end{array}\right]=\left[\begin{array}{c}
q_{1} \\
\vdots \\
q_{n} \\
q_{a}
\end{array}\right]_{(n+1) \times 1} \text {, }
\end{aligned}
$$

$$
\begin{aligned}
& \mathbf{T}_{g s}=\left[\begin{array}{c}
\mathbf{T}_{g} \\
-m_{a}
\end{array}\right]=-\mathbf{M}_{s}\left\{\begin{array}{c}
1 \\
\vdots \\
1 \\
1
\end{array}\right\}_{(n+1) \times 1}, \\
& \mathbf{T}_{u}=\left[\begin{array}{c}
-\mathbf{T}_{u} \\
1
\end{array}\right]=\left\{\begin{array}{c}
0 \\
\vdots \\
0 \\
-1 \\
1
\end{array}\right\}_{(n+1) \times 1} \\
& \mathbf{K}_{s}=\left[\begin{array}{cc}
\mathbf{K}+\mathbf{T}_{u} k_{a} \mathbf{T}_{n} & -\mathbf{T}_{u} k_{a} \\
-k_{a} \mathbf{T}_{n} & k_{a}
\end{array}\right] \\
& =\left[\begin{array}{cccc}
k_{1}+k_{2} & -k_{2} & 0 & 0 \\
-k_{2} & \ddots & -k_{n} & 0 \\
0 & -k_{n} & k_{n}+k_{a} & -k_{a} \\
0 & 0 & -k_{a} & k_{a}
\end{array}\right]_{(n+1) \times(n+1)} \text {, } \\
& \mathbf{C}_{s}=\left[\begin{array}{cc}
\mathbf{C}+\mathbf{T}_{u} c_{a} \mathbf{T}_{n} & -\mathbf{T}_{u} c_{a} \\
-c_{a} \mathbf{T}_{n} & c_{a}
\end{array}\right] \\
& =\left[\begin{array}{cccc}
c_{1}+c_{2} & -c_{2} & 0 & 0 \\
-c_{2} & \ddots & -c_{n} & 0 \\
0 & -c_{n} & c_{n}+c_{a} & -c_{a} \\
0 & 0 & -c_{a} & c_{a}
\end{array}\right]_{(n+1) \times(n+1)} \text {, }
\end{aligned}
$$

where $m_{i}, c_{i}$, and $k_{i}$ are the $i$ th floor mass, damping, and stiffness. Equation (5) can be represented in state space as follows:

$$
\dot{\mathbf{X}}_{\mathbf{s}}(\mathbf{t})=\mathbf{A}_{\mathbf{s}} \mathbf{X}_{s}(t)+\mathbf{B}_{1 s} \ddot{q}_{g}(t)+\mathbf{B}_{2 s} F_{a}(t),
$$

where $\mathbf{A}_{s} \in \mathbb{R}^{2(n+1) \times 2(n+1)}, \mathbf{B}_{1 s} \in \mathbb{R}^{2(n+1) \times 1}$, and $\mathbf{B}_{2 s} \in$ $\mathbb{R}^{2(n+1) \times 1}$ are system matrix, excitation matrix, and control matrix, respectively.

One can see that

$$
\begin{gathered}
\mathbf{A}_{s}=\left[\begin{array}{cc}
{[\mathbf{0}]_{(n+1) \times(n+1)}} & {[\mathbf{I}]_{(n+1) \times(n+1)}} \\
-\mathbf{M}_{s}^{-1} \mathbf{K}_{s} & -\mathbf{M}_{s}^{-1} \mathbf{C}_{s}
\end{array}\right], \\
\mathbf{B}_{1 s}=\left[\begin{array}{c}
{[\mathbf{0}]_{(n+1) \times 1}} \\
\mathbf{M}_{s}^{-1} \mathbf{T}_{g s}
\end{array}\right], \\
\mathbf{B}_{2 s}=\left[\begin{array}{c}
{[\mathbf{0}]_{(n+1) \times 1}} \\
\mathbf{M}_{s}^{-1} \mathbf{T}_{u s}
\end{array}\right] .
\end{gathered}
$$




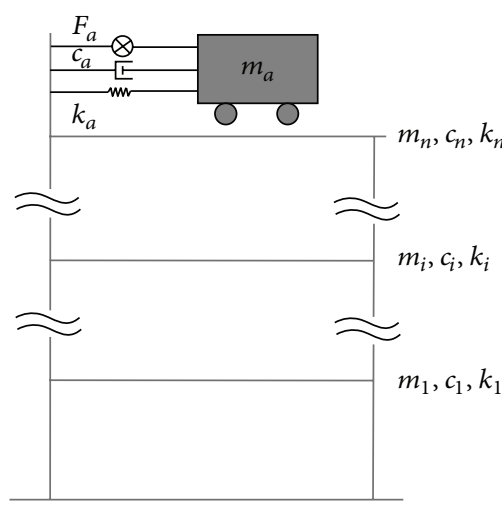

(a)

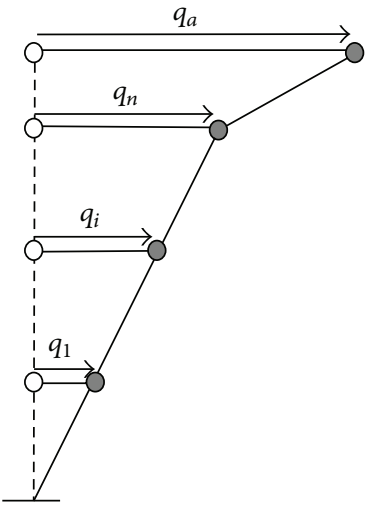

(b)

FIGURE 1: The model of AMD structure system.

$\mathbf{X}_{s}(t)=\left[\mathbf{q}_{s}(t) ; \dot{\mathbf{q}}_{s}(t)\right] \in \mathbb{R}^{2(n+1) \times 1}$ is the state vector, which contains relative displacement and relative velocity. This relative state vector can be transformed to another state vector which consists of interstory drift and interstory velocity as,

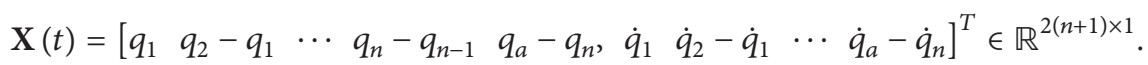

Define a transformation matrix $\Gamma \in \mathbb{R}^{2(n+1) \times 2(n+1)}$ to obtain $\mathbf{X}(t)$ such that $\mathbf{X}(t)=\Gamma \mathbf{X}_{s}(t)$. Substituting $\mathbf{X}_{s}(t)=$ $\Gamma^{-1} \mathbf{X}(t)$ into (7) and left-multiplying this equation with $\Gamma$, the state equation expressed by $\mathbf{X}(t)$ becomes

$$
\dot{\mathbf{X}}(t)=\mathbf{A X}(t)+\mathbf{B}_{1} \ddot{q}_{g}(t)+\mathbf{B}_{2} F_{a}(t),
$$

where $\mathbf{A}=\mathbf{\Gamma} \mathbf{A}_{s} \boldsymbol{\Gamma}^{-1} \in \mathbb{R}^{2(n+1) \times 2(n+1)}$, and $\mathbf{B}_{1}=\Gamma \mathbf{B}_{1 s} \in$ $\mathbb{R}^{2(n+1) \times 1}, \mathbf{B}_{2}=\mathbf{\Gamma} \mathbf{B}_{2 s} \in \mathbb{R}^{2(n+1) \times 1}$, and $\boldsymbol{\Gamma}$ is shown as

$$
\begin{aligned}
\boldsymbol{\Gamma} & =\left[\begin{array}{ccc}
\boldsymbol{\Gamma}_{s} & & {[\mathbf{0}]_{(n+1) \times(n+1)}} \\
{[\mathbf{0}]_{(n+1) \times(n+1)}} & & \boldsymbol{\Gamma}_{s}
\end{array}\right], \\
\boldsymbol{\Gamma}_{s} & {\left[\begin{array}{ccccc}
1 & 0 & \cdots & \cdots & 0 \\
-1 & 1 & 0 & \cdots & 0 \\
0 & \ddots & \ddots & & \vdots \\
\vdots & & \ddots & \ddots & 0 \\
0 & \cdots & \cdots & -1 & 1
\end{array}\right]_{(n+1) \times(n+1)} }
\end{aligned}
$$

\section{Fault Detection and Isolation and Fault Tolerant Control}

A simple and efficient way to design a controller for this structural control system with AMD is to design the Ho state feedback gain $\mathbf{G}_{\infty} \in \mathbb{R}^{1 \times 2(n+1)}$ [24], such that

$$
F_{a}(t)=\mathbf{G}_{\infty} \mathbf{X}(t) .
$$

However, when there are some faults in sensors or actuators, the performance of vibration suppressing may be degraded and even enlarge the structural response. So, it is necessary to detect and isolate faults such that the controller can tolerate the faults. This paper only considers the faults in sensors and designs a fault detection and isolation (FDI) filter. The designed FDI filter is intended to estimate structural system state, which can be used to reappear the structural sensor data with fewer faults. The difference between the measured output data and the estimated output data is the faults. The estimated output data can be used to isolate the faults transmitted to the designed Ho state feedback gain $\mathbf{G}_{\infty}$. So, the controller strategy is reformed and can perform the fault tolerant control performance.

For the faults in sensors, the sensor data including faults can be represented as

$$
\mathbf{y}_{v}(t)=\mathbf{C}_{y} \mathbf{X}(t)+\mathbf{v}(t),
$$

where $\mathbf{v}(t) \in \mathbb{R}^{q \times 1}$ is the faults part in sensor data $\mathbf{y}_{v}(t) \epsilon$ $\mathbb{R}^{q \times 1}$ and $\mathbf{C}_{y} \mathbf{X}(t)$ is the real part of sensor data, which is the system real output. The faults can be recognized as the additional data into the real sensor data. Combined with (10), the following equation can be derived:

$$
\begin{gathered}
\dot{\mathbf{X}}(t)=\mathbf{A X}(t)+\mathbf{B}_{1} \ddot{q}_{g}(t)+\mathbf{B}_{2} F_{a}(t), \\
\mathbf{y}_{v}(t)=\mathbf{C}_{y} \mathbf{X}(t)+\mathbf{v}(t) .
\end{gathered}
$$

The faults estimator is shown in Figure 2 and its state space formulation can be described as

$$
\begin{gathered}
\dot{\hat{\mathbf{X}}}(t)=\mathbf{A} \widehat{\mathbf{X}}(t)+\mathbf{B}_{2} F_{a}(t)+\mathbf{r}(t), \\
\widehat{\mathbf{y}}(t)=\mathbf{C}_{y} \widehat{\mathbf{X}}(t), \\
\mathbf{r}(t)=\mathbf{K}\left(\widehat{\mathbf{y}}(t)-\mathbf{y}_{v}(t)\right),
\end{gathered}
$$




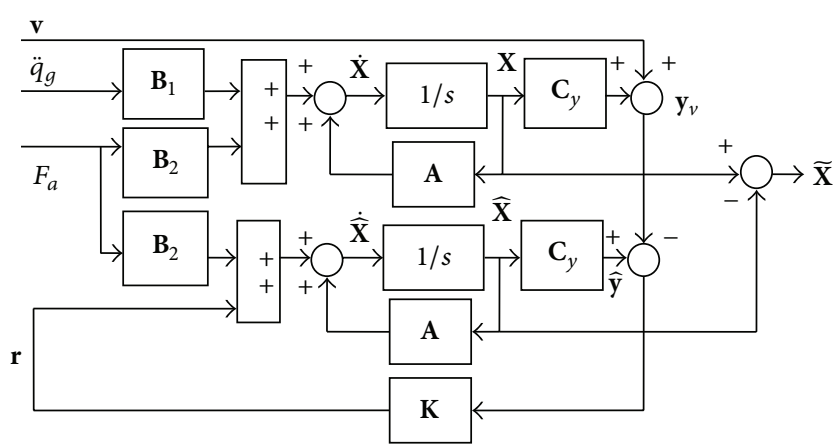

FIGURE 2: FDI diagram of AMD structure system.

where $\widehat{\mathbf{X}}(t) \in \mathbb{R}^{2(n+1) \times 1}$ is the estimated state for the structural state $\mathbf{X}(t), \widehat{\mathbf{y}}(t) \in \mathbb{R}^{q \times 1}$ is the estimated measurement output for the real part of $\mathbf{y}_{v}(t) \in \mathbb{R}^{q \times 1}$, that is, $\mathbf{C}_{y} \mathbf{X}(t), \mathbf{r}(t) \epsilon$ $\mathbb{R}^{2(n+1) \times 1}$ is the adjustment vector for estimated state vector, and $\mathbf{K} \in \mathbb{R}^{2(n+1) \times q}$ is the FDI filter to make the estimated state vector closed with structural system state vector. In this structural system with the sensor faults, the state is estimated so that the sensor data real part $\mathbf{C}_{y} \mathbf{X}(t)$ can be estimated as $\widehat{\mathbf{y}}(t)=\mathbf{C}_{y} \widehat{\mathbf{X}}(t)$. The faults in sensor data $\mathbf{y}_{v}(t) \in \mathbb{R}^{q \times 1}$ can be calculated by $\mathbf{y}_{v}(t)-\widehat{\mathbf{y}}(t)$ and then can be isolated by replacing $\mathbf{y}_{v}(t)$ with $\widehat{\mathbf{y}}(t)$. This is the fault detection and isolation (FDI). According to (14) and (15), the residue equation is obtained as follows:

$$
\begin{aligned}
& \dot{\widetilde{\mathbf{X}}}(t)=\widetilde{\mathbf{A}} \widetilde{\mathbf{X}}(t)+\widetilde{\mathbf{B}} \mathbf{w}(t), \\
& \widetilde{\mathbf{Z}}(t)=\widetilde{\mathbf{C}} \widetilde{\mathbf{X}}(t)+\widetilde{\mathbf{D}} \mathbf{w}(t) .
\end{aligned}
$$

$\widetilde{\mathbf{A}}, \widetilde{\mathbf{B}}, \widetilde{\mathbf{C}}, \widetilde{\mathbf{D}}, \widetilde{\mathbf{X}}(t), \widetilde{\mathbf{Z}}(t)$, and $\mathbf{w}(t)$ are defined as

$$
\begin{gathered}
\widetilde{\mathbf{A}}=\mathbf{A}+\mathbf{K C}_{y} \in \mathbb{R}^{2(n+1) \times 2(n+1)}, \\
\widetilde{\mathbf{B}}=\left[\begin{array}{ll}
\mathbf{B}_{1} & \mathbf{K}
\end{array}\right] \in \mathbb{R}^{2(n+1) \times(q+1)},
\end{gathered}
$$

$$
\begin{gathered}
\widetilde{\mathbf{C}}=\mathbf{I} \in \mathbb{R}^{2(n+1) \times 2(n+1)}, \\
\widetilde{\mathbf{D}}=[\mathbf{0}]_{2(n+1) \times(q+1)} \in \mathbb{R}^{2(n+1) \times(q+1)}, \\
\widetilde{\mathbf{X}}(t)=(\mathbf{X}(t)-\widehat{\mathbf{X}}(t)) \in \mathbb{R}^{2(n+1) \times 1}, \\
\widetilde{\mathbf{Z}}(t)=\widetilde{\mathbf{X}}(t) \in \mathbb{R}^{2(n+1) \times 1}, \\
\mathbf{w}(t)=\left[\ddot{q}_{g}(t) ; \mathbf{v}(t)\right] \in \mathbb{R}^{(q+1) \times 1},
\end{gathered}
$$

where $\widetilde{\mathbf{Z}}(t)=\widetilde{\mathbf{X}}(t) \in \mathbb{R}^{2(n+1) \times 1}$ is the output of the residue equation and $\widetilde{\mathbf{X}}(t)$ is the state of the residue equation.

In order to estimate the system state $\mathbf{X}(t)$ precisely, this paper designs the FDI filter $\mathbf{K}$ in (15) to minimize the Ho norm of the transfer function from input $\mathbf{w}(t)$ to output $\widetilde{\mathbf{Z}}(t)$. This design can be recognized as Ho optimization problem. It can be described as designing a static controller K to stabilize the system (16) such that

$$
J=\left\|\mathbf{H}_{\mathbf{z w}}(s)\right\|_{\infty}=\sup _{\omega,\|\omega(t)\|_{2} \neq 0}\left(\frac{\|\tilde{\mathbf{Z}}(t)\|_{2}}{\|\mathbf{w}(t)\|_{2}}\right)<\gamma,
$$

where $\gamma$ is a given scalar. According to the bounded real lemma [25], the following two statements are equivalent:

(1) Ho norm of the system in (16) is less than $\gamma$, and $\widetilde{\mathbf{A}}$ is stable;

(2) There exists a symmetric positive definite matrix $\Phi \in \mathbb{R}^{2(n+1) \times 2(n+1)}$ such that the following matrix inequality holds:

$$
\left[\begin{array}{ccc}
\widetilde{\mathbf{A}}^{T} \boldsymbol{\Phi}+\boldsymbol{\Phi} \widetilde{\mathbf{A}} & \boldsymbol{\Phi} \widetilde{\mathbf{B}} & \widetilde{\mathbf{C}}^{T} \\
\widetilde{\mathbf{B}}^{T} \boldsymbol{\Phi} & -\gamma \mathbf{I} & \widetilde{\mathbf{D}}^{T} \\
\widetilde{\mathbf{C}} & \widetilde{\mathbf{D}} & -\gamma \mathbf{I}
\end{array}\right]<\mathbf{0} .
$$

Substituting (17b) into (19),

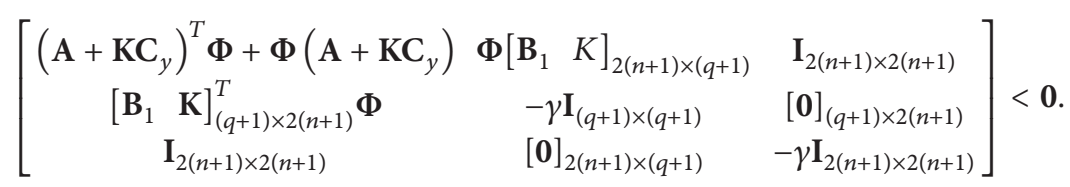

Because both $\boldsymbol{\Phi}$ and $\mathbf{K}$ are unknown variables and multiplied together, this problem cannot be solved directly. This paper employs variable substitution method [23] to linearize this constraint.
Define a new variable $\boldsymbol{\Xi}=\boldsymbol{\Phi} \mathbf{K} \in \mathbb{R}^{2(n+1) \times q}$ and take it into (20)

$$
\left[\begin{array}{cccc}
\mathbf{A}^{T} \boldsymbol{\Phi}+\boldsymbol{\Phi} \mathbf{A}+\mathbf{C}_{y}^{T} \mathbf{\Xi}^{T}+\boldsymbol{\Xi C}_{y} & {\left[\mathbf{\Phi} \mathbf{B}_{1}\right]_{2(n+1) \times 1}} & \boldsymbol{\Xi}_{2(n+1) \times q} & \mathbf{I}_{2(n+1) \times 2(n+1)} \\
{\left[\mathbf{B}_{1}^{T} \boldsymbol{\Phi}\right]_{1 \times 2(n+1)}} & -\gamma \mathbf{I}_{1 \times 1} & {[\mathbf{0}]_{1 \times q}} & {[\mathbf{0}]_{1 \times 2(n+1)}} \\
\boldsymbol{\Xi}_{q \times 2(n+1)} & {[\mathbf{0}]_{q \times 1}} & -\gamma \mathbf{I}_{q \times q} & {[\mathbf{0}]_{q \times 2(n+1)}} \\
\mathbf{I}_{2(n+1) \times 2(n+1)} & {[\mathbf{0}]_{2(n+1) \times 1}} & {[\mathbf{0}]_{2(n+1) \times q}} & -\gamma \mathbf{I}_{2(n+1) \times 2(n+1)}
\end{array}\right]<\mathbf{0} .
$$


Equation (21) is a linear matrix inequality with matrix variables $\boldsymbol{\Phi}$ and $\boldsymbol{\Xi}$. The optimal values of $\boldsymbol{\Phi}$ and $\boldsymbol{\Xi}$ can be computed through the solver feasp and mincx in MATLAB LMI toolbox. Then, $\mathbf{K}$ can be obtained from $\mathbf{K}=\boldsymbol{\Phi}^{-1} \boldsymbol{\Xi}$. So, the FDI filter in (15) is designed.

Through FDI filter, the estimated state $\widehat{\mathbf{X}}(t)$ and the estimated sensor data $\widehat{\mathbf{y}}(t)=\mathbf{C}_{y} \widehat{\mathbf{X}}(t)$ can be very closed to the system state $\mathbf{X}(t)$ and the sensor data real part $\mathbf{C}_{y} \mathbf{X}(t)$, respectively. The estimated state $\widehat{\mathbf{X}}(t)$ can be recognized as the input of $\mathrm{H} \infty$ state feedback gain $\mathbf{G}_{\infty}$. This reformed control system can be described in Figure 3. The reformed control strategy is different from the one in (12)

$$
\bar{F}_{a}(t)=\mathbf{G}_{\infty} \widehat{\mathbf{X}}(t) .
$$

As a summary, when the sensor data has faults, the control system cannot guarantee a good performance. Through FDI filter, the sensor data real part and system state can be estimated. The estimated information can be used to isolate the sensor faults. Then, put the estimated state into the previous designed Ho state feedback controller to reform the control strategy such that the control system can perform the fault tolerant control (FTC).

\section{Numerical Example}

Considering a three-story numerical example [26], an AMD control device is set on the top of this structure as shown in
Figure 4. Define the structural mass, damping, and stiffness as follows:

$$
\begin{gathered}
\mathbf{M}=\left[\begin{array}{lll}
6 & & \\
& 6 & \\
& & 6
\end{array}\right] \times 10^{3} \mathrm{~kg}, \\
\mathbf{C}=\left[\begin{array}{ccc}
12.4 & -5.16 & \\
-5.16 & 12.4 & -4.59 \\
& -4.59 & 7.2
\end{array}\right] \times 10^{3} \mathrm{~N} /(\mathrm{m} / \mathrm{s}), \\
\mathbf{K}=\left[\begin{array}{ccc}
3.4 & -1.8 & \\
-1.8 & 3.4 & -1.6 \\
& -1.6 & 1.6
\end{array}\right] \times 10^{6} \mathrm{~N} / \mathrm{m} .
\end{gathered}
$$

AMD control system mass, damping, and stiffness are as follows:

$$
\begin{gathered}
m_{a}=360 \mathrm{~kg}, \\
k_{a}=18819 \mathrm{~N} / \mathrm{m}, \\
c_{a}=365.3910 \mathrm{~N} /(\mathrm{m} / \mathrm{s}) .
\end{gathered}
$$

Suppose the sensor measurement outputs are the system states. Ho state feedback control strategy is employed to control this structural system with AMD. The Ho state feedback gain $\mathbf{G}_{\infty}$ in (12) can be obtained as follows:

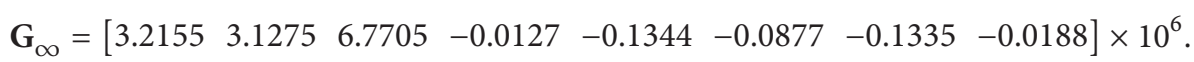

The El Centro NS earthquake acceleration record $\ddot{q}_{g}(t)$ with its peak scaled to $200 \mathrm{gal}\left(2 \mathrm{~m} / \mathrm{s}^{2}\right)$ is used as the ground excitation. The response suppression comparisons are shown in Figures 5 and 6 for the bare structure without control, passive tuned mass damper (TMD) structure, and active mass damper (AMD) structure without sensor faults. The bare structure is the structure without actuators. The TMD structure has actuators but does not generate control force. The control strategy for the AMD structure is Ho state feedback control.

In Figure 6, $\mathbf{G}_{\infty}$ represents Ho state feedback controller. Figures 5 and 6 illustrate the relative displacement response on the 3rd floor. The maximum value of the relative displacement for the TMD structure is $24.1 \%$ less than the one for the bare structure, while AMD structure is $67.0 \%$ off. Therefore,

$$
\mathbf{K}=\left[\begin{array}{cccc}
-6.4162 & -2.4851 & -0.9160 & 0.4926 \\
-1.0675 & -2.3501 & -0.6025 & 0.1106 \\
0.3623 & -0.1068 & -1.8490 & -0.4097
\end{array}\right.
$$

Taking $\mathbf{K}$ into (15) and putting the estimated state $\widehat{\mathbf{X}}(t)$ into state feedback gain $\mathbf{G}_{\infty}$, the control strategy is reformed to perform the fault tolerate control.
TMD and AMD can both suppress the structural vibration. Furthermore, AMD structure can reduce the vibration $56.5 \%$ more than TMD structure.

If there exist some faults in sensors, the faulty signal data $\mathbf{v}(t)$ is supposed to be located in the third floor displacement sensors. And its mean value and variance are zero and $2.5 \times$ $10^{-5}$, respectively. The comparison of the 3rd interstory drift between the AMD structure and the TMD structure is shown in Figure 7.

From Figure 7, AMD structural control system with Ho state feedback control strategy loses its control performance when there exist faults in the sensors. Accordingly, it is necessary to detect and isolate the faults in the sensors. Through proposed FDI filter design method, the gain $\mathbf{K}$ is obtained as follows:

$$
\left.\begin{array}{cccc}
-92.0527 & -118.6123 & -28.5338 & -23.9462 \\
-42.2075 & 93.6605 & -82.5947 & -27.4098 \\
39.9383 & -55.9578 & 105.6514 & -90.3240
\end{array}\right]^{T} \text {. }
$$

Figure 8 compares the interstory drift in the top story between the real interstory drift and the estimated drift. The related coefficients are shown in Table 1. From Table 1, the 


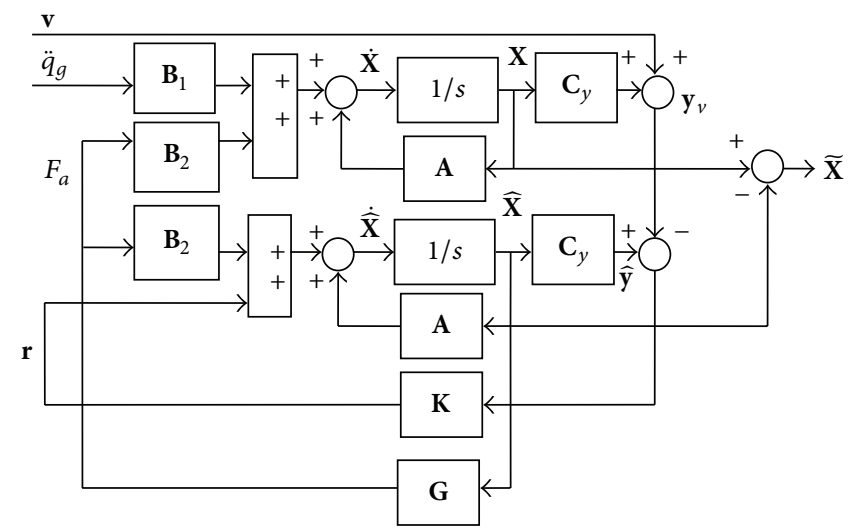

FIgURE 3: FTC diagram of AMD structure system.

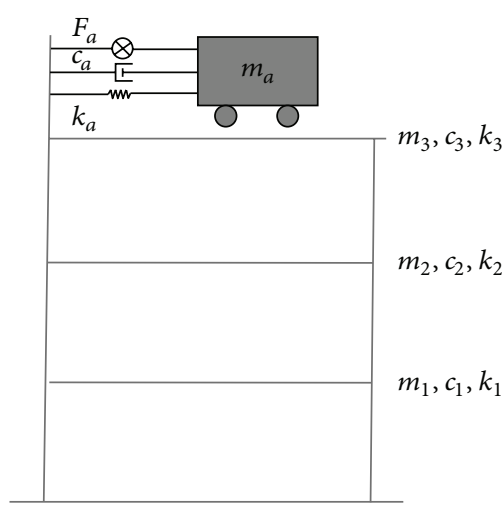

(a)

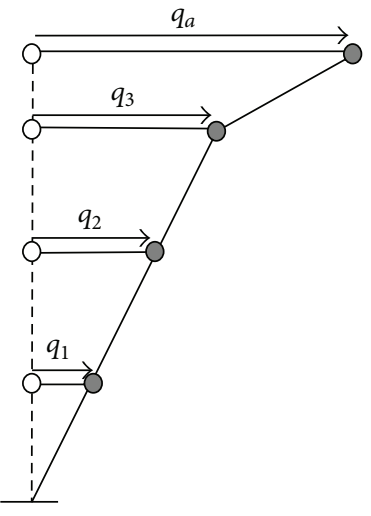

(b)

FIGURE 4: Three-story structure model with AMD system.

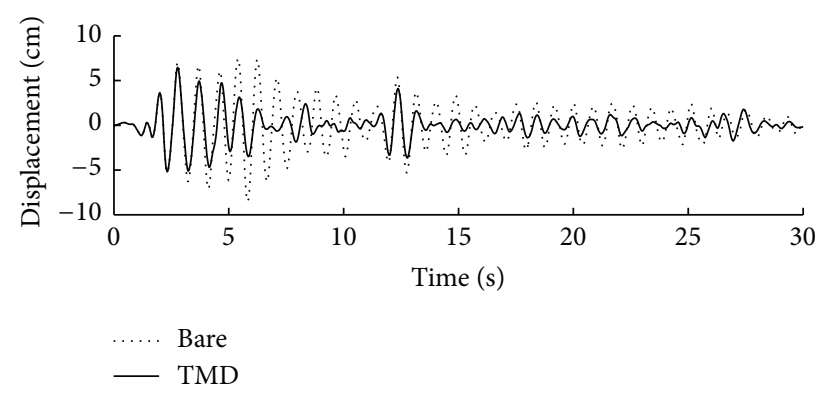

FIGURE 5: Comparison of the 3rd floor relative displacement between no control and TMD passive control.

dash line, which is the real drift, matches the solid line, which is the estimated drift by FDI. From Table 1, the related coefficients (RCs) are almost close to 1, which means that FDI can estimate drift precisely.

Through FTC strategy, the interstory drift is suppressed, and the third story drift is shown in Figures 9 and 10. Table 2 shows some details about vibration suppression effects for other stories.

Figure 9 illustrates the comparison of the interstory drift in the 3rd story between TMD structure and AMD structure considering sensor faults. In AMD structure, the control

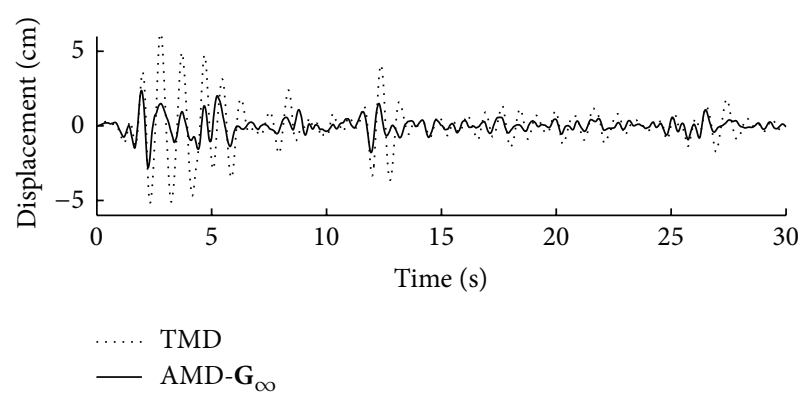

FIgURE 6: Comparison of the 3rd floor relative displacement between TMD passive control and AMD-Ho state feedback control.

strategy is FTC. AMD structure can suppress the structural vibration more effectively than TMD structure does. While only using Hoo state feedback control strategy, the vibration is worsened as shown in Figure 7. Figure 10 can illustrate the difference more directly.

From Table 2, the maximum values of all the interstory drifts for AMD structure with Ho state feedback control strategy are $29.37 \%, 38.29 \%$, and $-46.50 \%$ less than the TMD structural drifts, respectively. While using FTC strategy, the drifts are $23.42 \%, 39.19 \%$, and $54.78 \%$ less than the TMD 
TABLE 1: Correlation coefficient between FDI estimated state and the real state.

\section{Comparison between the states estimated by FDI and structure system real states} Interstory drift

Interstory velocity

\begin{tabular}{cccccccc} 
& $\begin{array}{c}q_{1} \\
\mathrm{~cm}\end{array}$ & $\begin{array}{c}q_{2}-q_{1} \\
\mathrm{~cm}\end{array}$ & $\begin{array}{c}q_{3}-q_{2} \\
\mathrm{~cm}\end{array}$ & $\begin{array}{c}q_{\mathrm{a}}-q_{3} \\
\mathrm{~cm}\end{array}$ & $\begin{array}{c}\dot{q}_{1} \\
\mathrm{~cm}\end{array}$ & $\begin{array}{c}\dot{q}_{2}-\dot{q}_{1} \\
\mathrm{~cm}\end{array}$ & $\begin{array}{c}\dot{q}_{3}-\dot{q}_{2} \\
\mathrm{~cm}\end{array}$ \\
\hline $\mathrm{RC}$ & 0.8422 & 0.8064 & 0.8584 & 0.9989 & 0.7741 & 0.7483 & 0.9035 \\
\hline
\end{tabular}

TABLE 2: Suppressing effect comparisons among the bared structure, TMD structure, AMD structure without faults, and AMD Ho structure with faults.

\begin{tabular}{|c|c|c|c|c|c|}
\hline Systems & $\begin{array}{l}q_{1} \\
\mathrm{~cm}\end{array}$ & $\begin{array}{c}q_{2}-q_{1} \\
\mathrm{~cm}\end{array}$ & $\begin{array}{c}q_{3}-q_{2} \\
\mathrm{~cm}\end{array}$ & $\begin{array}{c}q_{\mathrm{a}}-q_{3} \\
\mathrm{~cm}\end{array}$ & $\begin{array}{c}F_{a}(t) \\
\mathrm{kN}\end{array}$ \\
\hline Bare system & 4.04 & 2.81 & 1.68 & - & - \\
\hline TMD system & 2.69 & 2.22 & 1.57 & 25.08 & - \\
\hline AMD system without faults using $\mathbf{G}_{\infty}$ & 1.88 & 1.08 & 0.86 & 78.38 & 15.46 \\
\hline AMD system with faults using $\mathbf{G}_{\infty}$ & 1.90 & 1.37 & 2.30 & 108.7 & 209.1 \\
\hline AMD system with faults using FTC & 2.06 & 1.35 & 0.71 & 83.36 & 16.00 \\
\hline
\end{tabular}

$\mathbf{G}_{\infty}$ represents $\mathrm{H} \infty$ state feedback control.

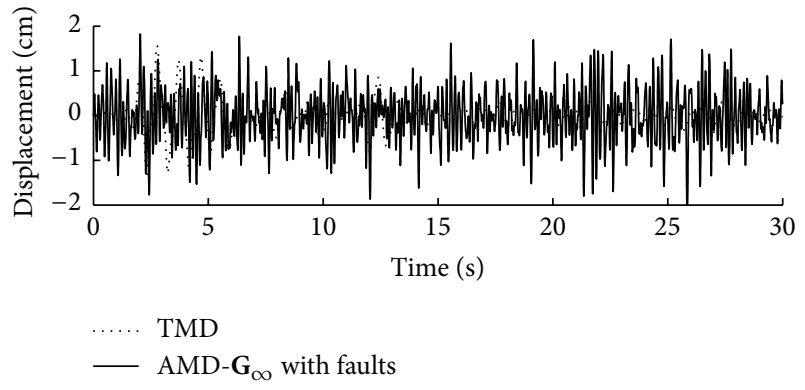

Figure 7: Comparison of the 3rd story interstory drift between TMD and AMD with Ho state feedback control strategy considering the sensor faults.

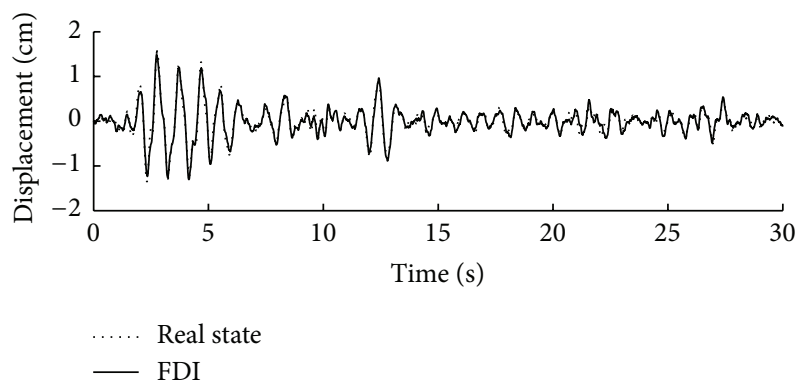

FIGURE 8: Comparison between the real and FDI estimated interstory drift in the top story.

structural drifts, respectively. The actuator force $F_{a}(t)$ saves a lot of energy using FTC strategy than when only using Ho state feedback control strategy. Therefore, FTC can tolerate faults in sensors and suppress structural vibration efficiently.

\section{Conclusions}

In practical engineering, structural control system may have faults which can degrade control system performance. This

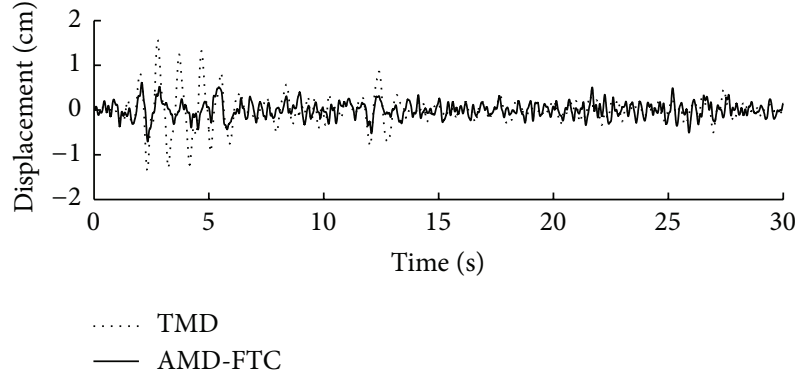

FIGURE 9: Comparison of the interstory drifts in the 3rd story between the TMD and AMD-FTC control.

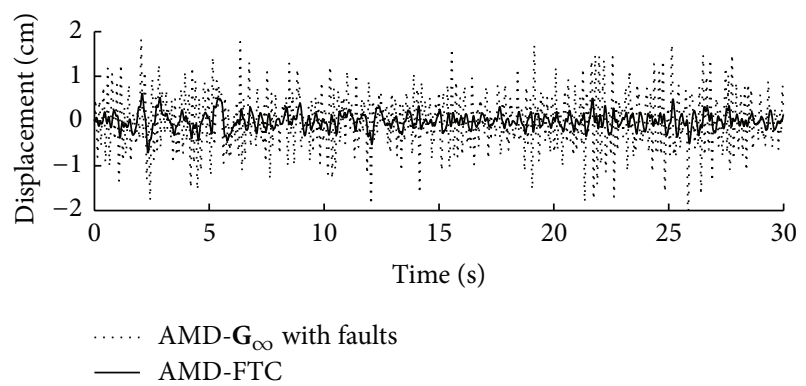

FIGURE 10: Comparison of the 3rd floor interstory drifts between the AMD-Ho state feedback control and AMD-FTC control.

paper presents fault tolerant control (FTC) for civil structures. Considering the faults in sensors, fault detection and isolation (FDI) filter is designed. Transmitting the estimated state to Ho state feedback controller designed previously, the reformed control strategy is obtained, which is called fault tolerant control (FTC). This paper also presents the derivation of FDI, which transforms the inequality matrix to linear inequality matrix using variable substitution method. A three-story numerical example validates FDI and FTC performance. From this study, there are some conclusions. 
(1) When there is no fault in sensors, Ho state feedback control in AMD structure has better suppressing performance than TMD structure.

(2) When there are faults in sensors, Ho state feedback control in AMD structure cannot guarantee the control performance and even worsen the vibration.

(3) FDI designed by the proposed method can detect and isolate the faults. It can estimate the system state precisely. According to the estimated state, the measurement output from sensors can also be estimated.

(4) FTC designed by the proposed method can tolerate the faults in sensors. So, it can strengthen the robustness of the control system.

\section{Acknowledgment}

The authors are grateful for the support of the National Natural Science Foundation of China (Grant nos. 51121005 and 51261120375).

\section{References}

[1] T. T. Soong, Active Structure Control Theory and Practice, Longman Scientific \& Technical, New York, NY, USA, 1990.

[2] T. T. Soong and G. F. Dargush, Passive Energy Dissipation Systems in Structural Engineering, John Wiley \& Sons, London, UK, 1997.

[3] J. T. P. Yao, "Concept of structural control," Journal of Structural Division, vol. 98, pp. 1567-1574, 1972.

[4] G. Song, P. Qiao, V. Sethi, and A. Prasad, "Active vibration control of a smart pultruded fiber-reinforced polymer I-beam," Smart Materials and Structures, vol. 13, no. 4, pp. 819-827, 2004.

[5] G. Song, S. P. Schmidt, and B. N. Agrawal, "Experimental study of active vibration suppression of flexible structure using modular control patch," in Proceedings of the IEEE Aerospace Conference, vol. 1, pp. 189-201, March 1998.

[6] G. Song, B. Kelly, and B. N. Agrawal, "Active position control of a shape memory alloy wire actuated composite beam," Smart Materials and Structures, vol. 9, no. 5, pp. 711-716, 2000.

[7] V. Sethi, M. A. Franchek, and G. Song, "Active multimodal vibration suppression of a flexible structure with piezoceramic sensor and actuator by using loop shaping," Journal of Vibration and Control, vol. 17, no. 13, pp. 1994-2006, 2011.

[8] V. Sethi and G. Song, "Multimodal vibration control of a flexible structure using piezoceramic sensor and actuator," Journal of Intelligent Material Systems and Structures, vol. 19, no. 5, pp. 573$582,2008$.

[9] H. Gu and G. Song, "Active vibration suppression of a flexible beam with piezoceramic patches using robust model reference control," Smart Materials and Structures, vol. 16, no. 4, pp. 14531459, 2007.

[10] L. Huo and H. Li, Multi-Dimensional Structure Damping Control, Science Press, Beijing, China, 2008.

[11] G. Song, B. Kelly, B. N. Agrawal, P. C. Lam, and T. S. Srivatsan, "Application of shape memory alloy wire actuator for precision position control of a composite beam," Journal of Materials Engineering and Performance, vol. 9, no. 3, pp. 330-333, 2000.

[12] Y. Wang, "Time-delayed dynamic output feedback $H_{\infty}$ controller design for civil structures: a decentralized approach through homotopic transformation," Structural Control and Health Monitoring, vol. 18, no. 2, pp. 121-139, 2011.

[13] Y. Bai and K. M. Grigoriadis, " $H_{\infty}$ collocated control of structural systems: an analytical bound approach," Journal of Guidance, Control, and Dynamics, vol. 28, no. 5, pp. 850-853, 2005.

[14] H. Gu, G. Song, and H. Malki, "Chattering-free fuzzy adaptive robust sliding-mode vibration control of a smart flexible beam," Smart Materials and Structures, vol. 17, no. 3, Article ID 035007, 2008.

[15] Y. Bai and K. M. Grigoriadis, "Damping parameter design optimization in structural systems using an explicit $H_{\infty}$ norm bound," Journal of Sound and Vibration, vol. 319, no. 3-5, pp. 795-806, 2009.

[16] H. Gu and G. Song, "Active vibration suppression of a composite I-beam using fuzzy positive position control," Smart Materials and Structures, vol. 14, no. 4, pp. 540-547, 2005.

[17] Y. Bai, K. M. Grigoriadis, and G. Song, "Active fault tolerant control of a flexible beam," in Modeling, Signal Processing, and Control for Smart Structures ., D. K. Lindner, Ed., vol. 6523 of Proceedings of SPIE, 2007.

[18] G. Song, L. Cai, Y. Wang, and R. W. Longman, "A slidingmode based smooth adaptive robust controller for friction compensation," International Journal of Robust and Nonlinear Control, vol. 8, no. 8, pp. 725-739, 1998.

[19] G. Song and N. Ma, "Robust control of a shape memory alloy wire actuated flap," Smart Materials and Structures, vol. 16, no. 6, pp. N51-N57, 2007.

[20] G. Song and R. Mukherjee, "A comparative study of conventional nonsmooth time-invariant and smooth time-varying robust compensators," IEEE Transactions on Control Systems Technology, vol. 6, no. 4, pp. 571-576, 1998.

[21] L. Li, G. Song, and J. Ou, "Adaptive fuzzy sliding mode based active vibration control of a smart beam with mass uncertainty," Structural Control and Health Monitoring, vol. 18, no. 1, pp. 4052, 2011.

[22] G. E. Stavroulakis, D. G. Marinova, and E. C. Zacharenakis, "Robust control in structural dynamics, taking into account structural uncertainties," in Proceedings of the 5th GRACM International Congress on Computational Mechanics, Limassol, Cyprus, 2005.

[23] L. Yu, Robust Control-Linear Matrix Inequalities Approach, Tsinghua University Press, Beijing, China, 2002.

[24] Y. Bai, K. M. Grigoriadis, and V. Sethi, "Static output feedback $H_{\infty}$ control of collocated structural systems," in Proceedings of the American Control Conference, vol. 1-12, pp. 828-833, June 2006.

[25] U. Mackenroth, Robust Control Systems: Theory and Case Studies, Springer, Berlin, Germany, 2004.

[26] Y. Wang, J. P. Lynch, and K. H. Law, "Decentralized $H_{\infty}$ controller design for large-scale civil structures," Earthquake Engineering and Structural Dynamics, vol. 38, no. 3, pp. 377-401, 2009. 


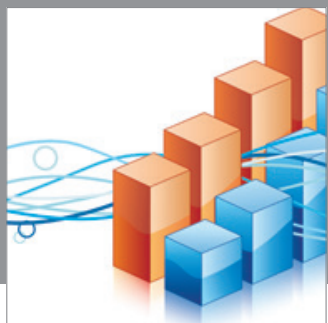

Advances in

Operations Research

mansans

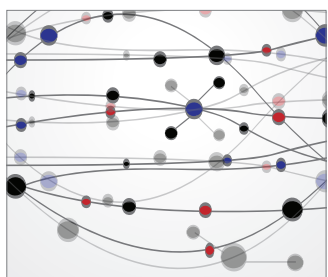

The Scientific World Journal
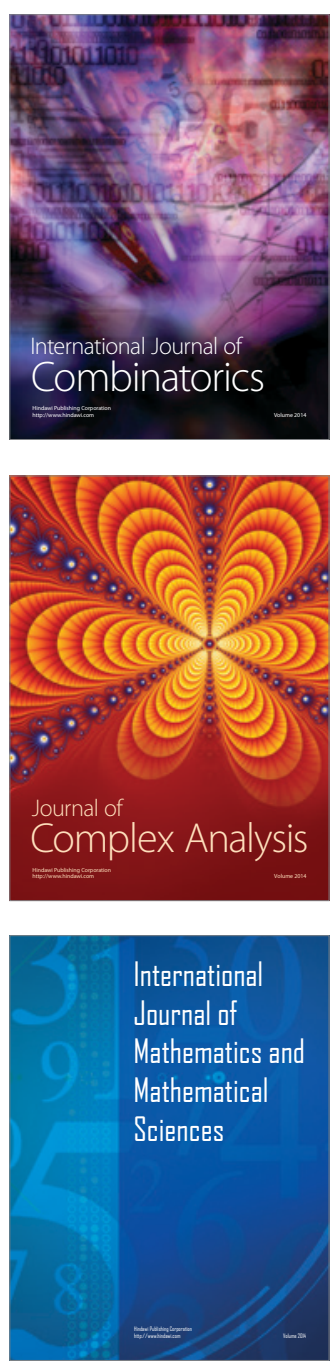
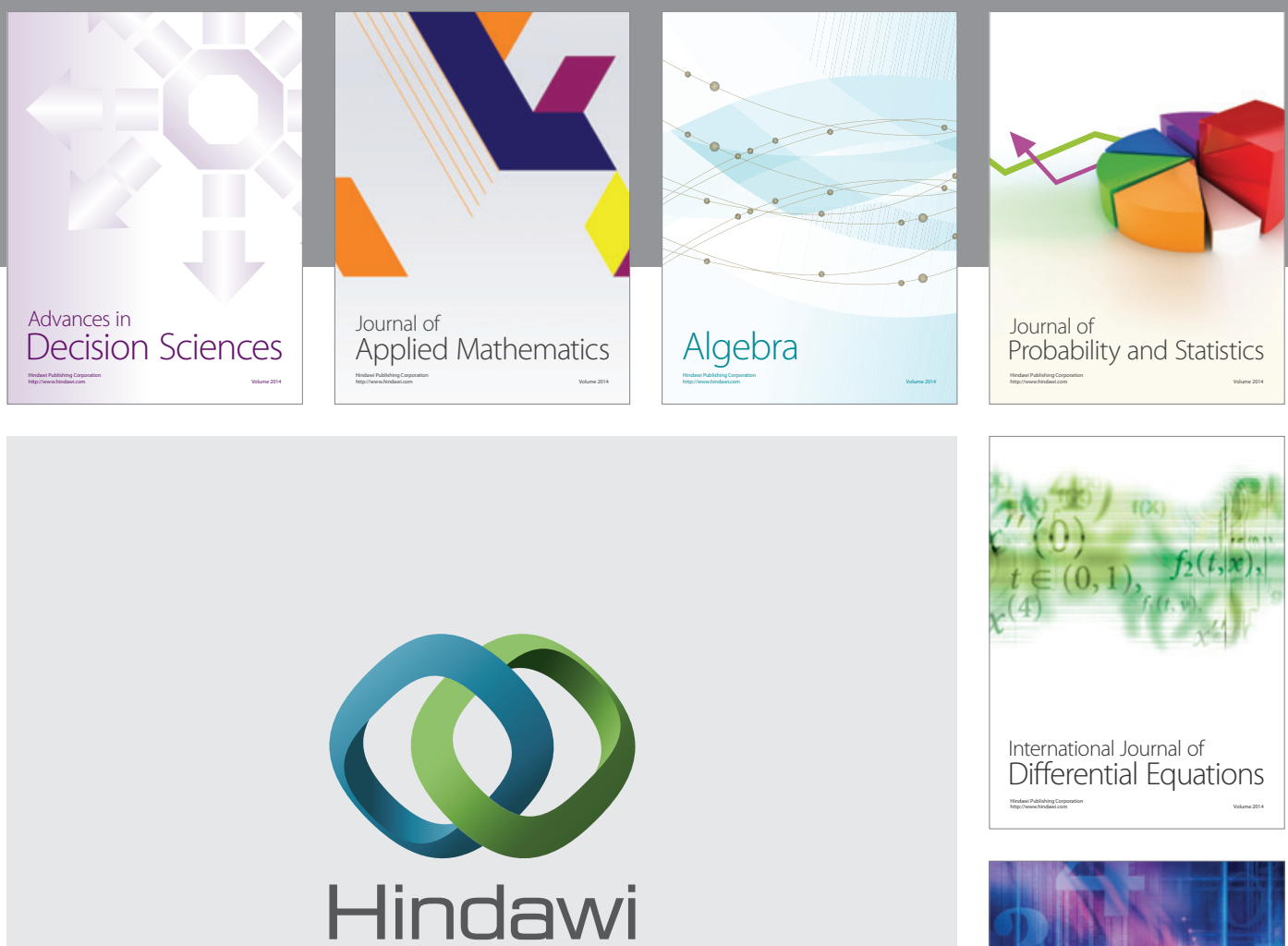

Submit your manuscripts at http://www.hindawi.com
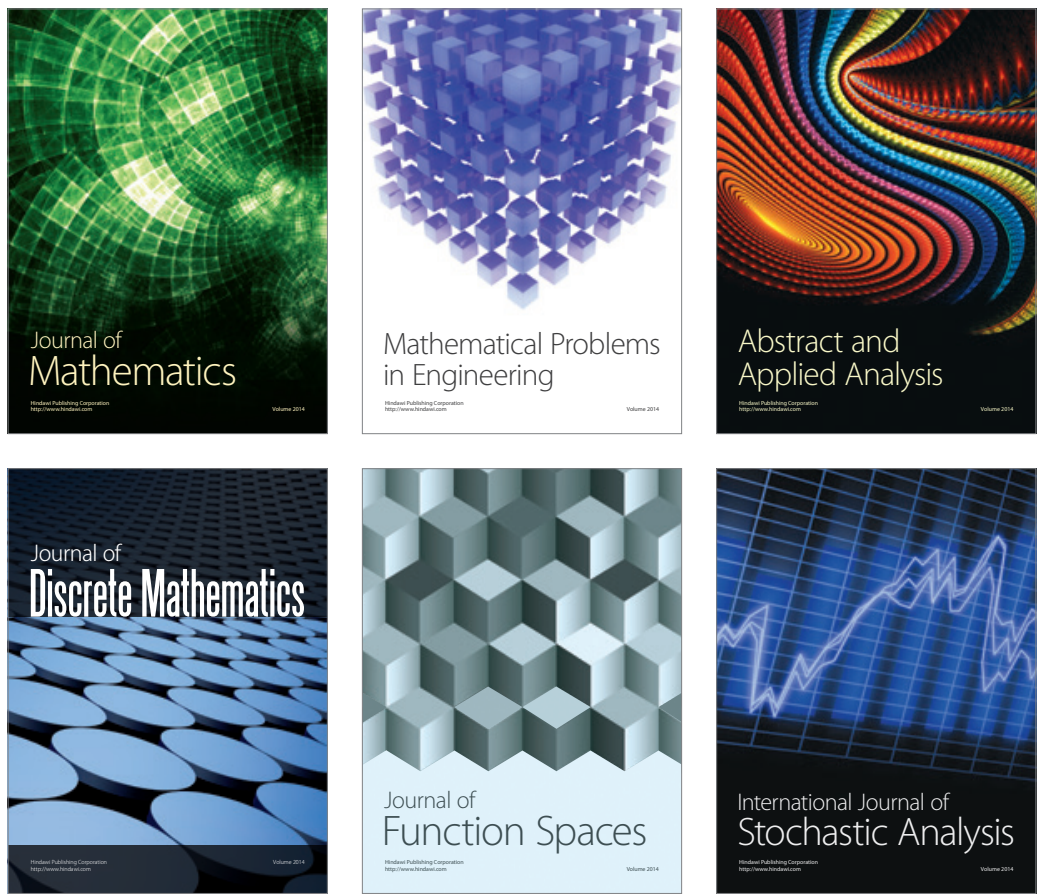

Journal of

Function Spaces

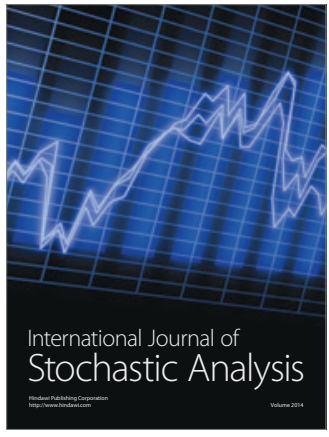

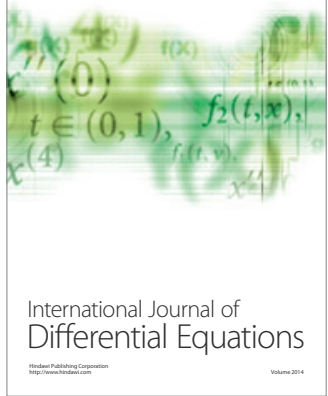
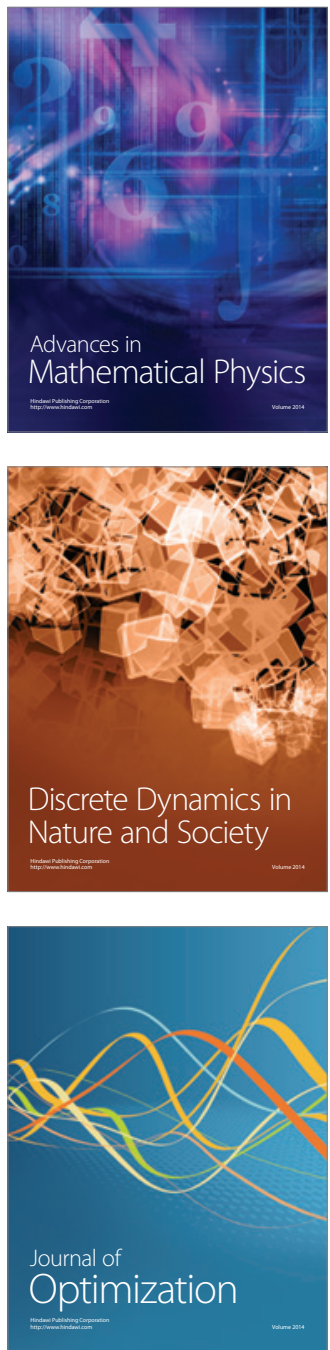